\title{
Effect of Curing Temperature and Curing Degree on Elastic Recovery of Conductive Particles
}

\author{
Min Gong \\ School of Electronical and Mechanical Engineering, \\ Guilin University of Electronic Technology \\ Guilin, 541004, China \\ Mzhnq123456@sina.cn
}

\author{
Dejian Zhou* \\ School of Electronical and Mechanical Engineering, \\ Guilin University of Electronic Technology \\ Guilin, 541004, China \\ Corresponding author: Emezdj@guet.edu.cn
}

\begin{abstract}
The nonlinear properties of elastic modulus of the resin in relation to curing degree of the resin was defined through the phenomenological model. The finite element model of LTCC-metal assembly, which belong to the radio frequency (RF) function layer, is established by ABAQUS. When curing pressure is determined, the effect of different curing degree on the elastic recovery of conductive particles is given under the same curing temperature, and the influence of different curing temperature on the deformation of conductive particle is also given under the same curing degree. Therefore, the curing degree increased is able to increase the contact area of conductive particles so as to reduce the contact resistance, but at the same time, the interconnection interface residual stress also increased, thus easy to cause the stratification. However, when the curing degree is determined, the contact area increased firstly, and then gradually decrease as the curing temperature increases, and when used in curing at lower temperature, the curing time increased accordingly, it will influence the economic benefits.
\end{abstract}

Keywords- the radio frequency (RF) function layer; elastic recovery of conductive particles; finite element modeling (FEM); anisotropic conductive adhesive (ACA); phenomenological model

\section{INTRODUCTION}

Low temperature co-firing ceramic(LTCC) as a new type of packaging technology, all kinds of passive devices in the circuit can be completely buried in the medium, and realized miniaturization of SMT products in the form of three dimensional structure of multilayer circuit, at the same time can be combined with active device used in developing all kinds of high integration, low cost, low power radio frequency and microwave modules[1]. At present, the ways of interconnecting between LTCC and metal which belong to the RF Function layer mainly have solder welding and adhesion. Older welding generally divided into tin-lead and lead-free soldering, because of its low cost, good wettability, long service life and high reliability, is widely used in microelectronic assembly. However, due to the inherent toxicity of lead and lead compounds, eliminate lead compounds is one of the important target in the next few years microelectronics industry[2]. Compared with the traditional solder welding, ACA (Anisotropic Conductive Adhesive) offers several advantages such as low temperatures, lead-free and simple processes. But the shortcomings are also obvious, such as the mechanical strength, conductive capability and stability of defects still exist.

So this paper combined with the actual research needs, and regarded structure-function integration components as the objects to research the influence rule of curing temperature and curing degree on elastic recovery of conductive particles in RF function layer ACA assembles. At present, the simulation analysis of ACA are mostly based on the assumption: adhesive is completely cured. But some research shows that there is the maximum dynamic shear strength when the curing degree of ACA is about $85 \%[3]$. In view of this, based on curing degree versus curing temperature and time, ABAQUS finite element software is used to establish FEM of LTCC-meter interconnection, and to research the effect of curing temperature and curing degree on the properties of conductive adhesive interconnection.
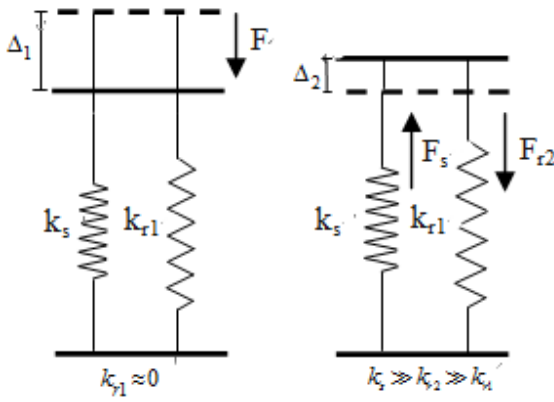

Figure 1. Simplified mechanics model

\section{RESEARCH APPROACHES}

In fact, the electrical connection is formed by connecting the adjacent conductive area in the upper and lower devices via the conductive particles in ACA assemblies, and the mechanical connection is realized through the cured adhesive[4]. As shown in Fig. 1, conductive particles and the cured resin simplified into springs[5]. Conductive adhesive interconnection process can be understood as conductive particles hot-pressing formed and rebound of conductive particles. In the course of rebound, $F_{s}$ as the resilience force of conductive particles, and $F_{r 2}$ as the resistance of elastic recovery of conductive particles. Therefore, it can be speculated that 
the rebound of conductive particles will impact the performance of ACA interconnection, and it obviously associated with curing degree.

In general, the conductive adhesive matrix is thermosetting epoxy resin, the curing reaction of resin is based on thermal activation of chemical reactions[6]. It's very common about researching cure kinetics of resin, there are two main types for model: phenomenological model and mechanism model. Phenomenological model is widely used in the numerical simulation and optimization design in the process of curing due to its simplicity[7], Phenomenological model based on the type (1):

$$
\frac{d \alpha}{d t}=A_{0} \exp \left[-\frac{E_{a}}{R T}\right](1-\alpha)^{n}
$$

Where $\alpha$ is curing degree, and $d \alpha / d t$ is the rate of cure, $R$ is the universal gas constant $E_{a}$ is the activation energy, $A$ is frequency factor, $T$ is the absolute temperature, and $n$ is order of the reaction. Based on DSC curve, $A, E_{a}$ and $n$ can be obtained by using Kissinger, Crane, and Arrhenius equations[8]. Then the curing degree changing with time can be obtained through the simulation calculation, as shown in Fig. 2.

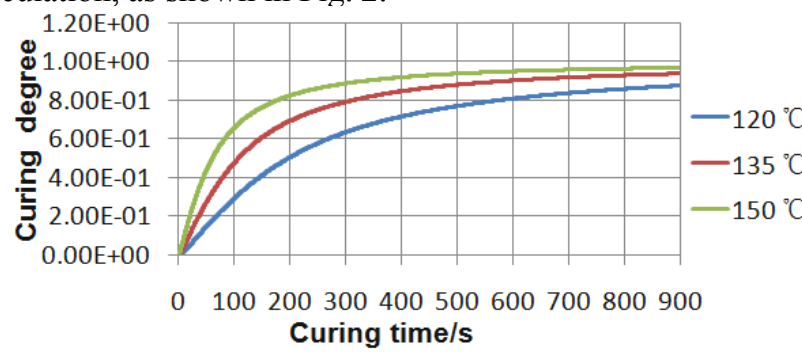

Figure 2. Curing degree versus time

\section{The Relation BetweEn Elasticity Modulus OF THE RESIN AND CURING DEGREE}

In the process of curing, the resin from low modulus, not curing state of viscous flow pattern transition to high modulus, completely curing state of the state. Elastic modulus of resin related to the curing process is determined by this type[9]:

$$
\begin{cases}E_{m}=E_{m}^{0} & \left(\alpha<\alpha_{g e l}\right) \\ E_{m}=\left(1-\alpha_{\text {mod }}\right) E_{m}^{0}+\alpha_{\text {mod }} E_{m}^{\infty} & \left(\alpha>\alpha_{\text {gel }}\right)\end{cases}
$$

Where $E_{m}$ is elastic modulus of resin, $E_{m}^{0}$ is elastic modulus of uncured resin, $E_{m}^{\infty}$ is elastic modulus of completely cured resin, $\alpha_{g e l}$ is gel point.

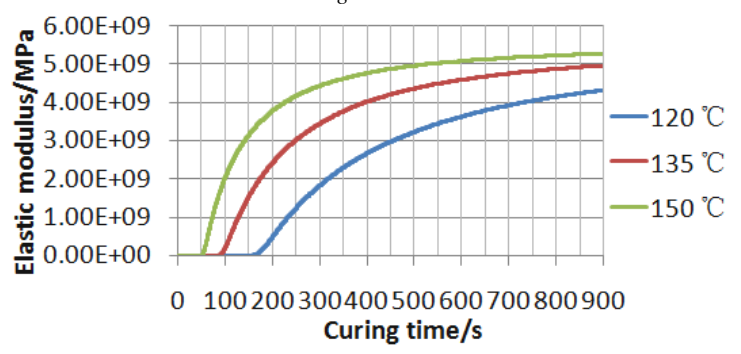

Figure 3. Elastic modulus vs curing time

The elastic modulus of resin is $5.577 \mathrm{GPa}$ when resin matrix of silver-epoxy adhesive completely cured, so $E_{m}^{0}$ is $5.577 \mathrm{MPa}, E_{m}^{\infty}$ is $5.577 \mathrm{GPa}$, and $\alpha_{\text {gel }}$ is 0.467 . By type (2) and (3) combined with the curve of Fig. 2 could get elastic modulus of resin changing with time under different temperature, as shown in Fig. 3.

\section{Finite ElEMENT SimUlations Styling}

\section{A. The finite element model and geometry}

In this research, the mathematical model of LTCC substrate layer does not have any components. The dimension of LTCC substrate was $30 \mathrm{~mm} \times 30 \mathrm{~mm} \times 3.175$ $\mathrm{mm}$ and height of gold-plated layer is $12 \mathrm{um}$, with its interconnection the dimension of metal substrate was 33 $\mathrm{mm} \times 33 \mathrm{~mm} \times 2 \mathrm{~mm}$ and height of gold-plated layer is 8 um. According to the previous theoretical analysis, conductive adhesive curing process simulation analysis is mainly divided into hot-press molding analysis of conductive particles and the rebound analysis of conductive particles. Compression molding analysis of conductive particles from the starting pressure conductive particles to conductive particles was out of shape. At this point, ACA continues to be in viscous flow state without force, So there is no resin in model of conductive particles compression, as shown in Fig. 4 on the right and up. Conductive particles springback simulation Model is formed by adding resin on the basis of the prior model, as shown in Fig. 4 on the right and down. In Fig. 4 on the left is the whole model.

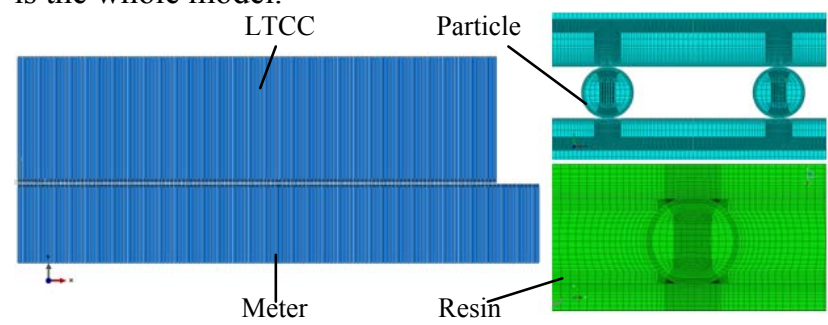

Figure 4. One-two geometry of 2D model and local FEM

\section{B. Material properties}

The finite element model is based on the following assumptions: 1.The key material gold-plated layer and conductive particles is elastic-plastic materials, the rest of material is elastic materials; 2 . Conductive particles evenly 
distributed ; 3 . Elasticity modulus of the resin relative to its curing degree, and thermal expansion coefficient is classified into two parts by glass transition temperature $T_{g}=80^{\circ} \mathrm{C}$, the value as shown in table 1 , other material properties have nothing to do with curing temperature and curing degree. Details are shown in table 1, and the unspecified units are SI units.

TABLE I. MATERIAL PROPERTIES

\begin{tabular}{|c|c|c|c|c|}
\hline Materials & $\mathbf{K}$ & $\begin{array}{c}\text { CTE } \\
{\left[10^{-6} K^{-1}\right]}\end{array}$ & $\begin{array}{c}\text { E } \\
\text { [GPa] }\end{array}$ & $\begin{array}{c}\sigma_{\mathrm{S}} \\
{[\mathrm{MPa}]}\end{array}$ \\
\hline Au & 298 & 14.4 & 77.2 & 105 \\
\hline ACA & 2.5 & $31.5 / 158$ & Fig.3 & I \\
\hline LTCC & 2 & 7 & 82 & 1 \\
\hline $\mathbf{T 2}$ & 397 & 16.7 & 120 & 1 \\
\hline \multirow[t]{2}{*}{ Ag } & 429 & 19.1 & 77 & 50 \\
\hline & $\mathbf{v}$ & $\rho$ & Cp & \\
\hline Au & 0.42 & 19000 & 132 & \\
\hline $\mathbf{A C A}$ & 0.3 & 2150 & 1100 & \\
\hline LTCC & 0.26 & 2500 & 729 & \\
\hline $\mathbf{T 2}$ & 0.3 & 8900 & 385 & \\
\hline Ag & 0.367 & 10500 & 237 & \\
\hline
\end{tabular}

\section{Loading conditions}

The outer surface of the packaging in the air environment and the temperature is $25^{\circ} \mathrm{C}$, the value of thermal transfer coefficient is $10 \mathrm{~W} /\left(\mathrm{m}^{2} \cdot \mathrm{K}\right)$. In the hotpress forming stage, temperature and pressure is provided by the hot-press head up and down. The heat transfer to the LTCC and metal layer to cure adhesive though heater surface. Therefore, on the up surface of the LTCC boundary conditions can be set to constant temperature conditions, the value of temperature is $T_{\text {top }}$; On the down surface of the metal the value of temperature can be defined $T_{b t m}$; The initial temperature of model can be set to $T_{0}=25^{\circ} \mathrm{C}$. The initial temperature of the process of particles rebound is $\left.T\right|_{t=0}=f(x, y, z)$, that is the temperature of the whole module when the thermal curing process is completed. After curing, the whole packaging parts in the natural cooling process, the upper and lower surfaces in a natural convection boundary conditions. In the hot-press molding analysis process for force analysis, the back of the LTCC bear the pressure of upper header, that $F_{X}=F_{Y}=0, F_{Z}=$ curing pressure; Lower header support the under surface of metal as the displacement boundary conditions. In the process of particles rebound, there is no outside force.

\section{RESUlTS AND DISCUSSION}

Finite element simulation, the elastic constitutive model is used to describe the resin matrix, and when curing temperature determined, the curing degree of changing over time, changing the elastic modulus of the resin, it makes the stress and strain of conductive adhesive interconnection interface more accurate, improve the ability of the simulation. This paper mainly studies the curing degree and temperature affect the performance of interconnection, so curing pressure is directly selected $5 \mathrm{MPa}$. Research shows that when the curing degree reaches more than $75 \%$ of the conductive adhesive is not easy to occur stratification, and the most outer layer conductive particles interconnect interface residual stress around the biggest[10]. So this article research institute in curing process parameters combination of curing degree were higher than $75 \%$, and extracting the value of the institute of all are the most outer layer conductive particles and the surrounding interconnect interface.

\section{A. The influence of curing degree of conductive particles rebound}

Fig. 2 shows that the curing degree of resin increases over time when the curing temperature is different. When the resin curing to a certain degree, Removed instant pressure conductive particles rebound will be affected by the curing degree. After cooling to room temperature, the interconnection of conductive adhesive performance will be different. As shown in Fig. 5(a): the stress diagram of conductive particles when the curing temperature is raised but not start curing. Fig. 5(b), (c) and (d) is show that the stress diagram of conductive particles after curing temperature is cooled to room temperature.

Conductive particles rebound will reduce conductive particle deformation, so do the stress and strain of conductive particles. As shown in Fig. 5, the amount of rebound of conductive particles decrease as the curing degree of resin increases. Through the simulation calculation, combined with Fig. 1, when $F_{s}$ is determined, the higher the degree of curing, the greater $F_{r 2}$, the greater the contact area of conductive particles in the end. Table 2 truthfully reflect the curing degree about the properties of conductive adhesive interconnection. When temperature determined, the higher the curing degree, the greater the contact area of conductive particles, as the results of the literature [5] and [10].
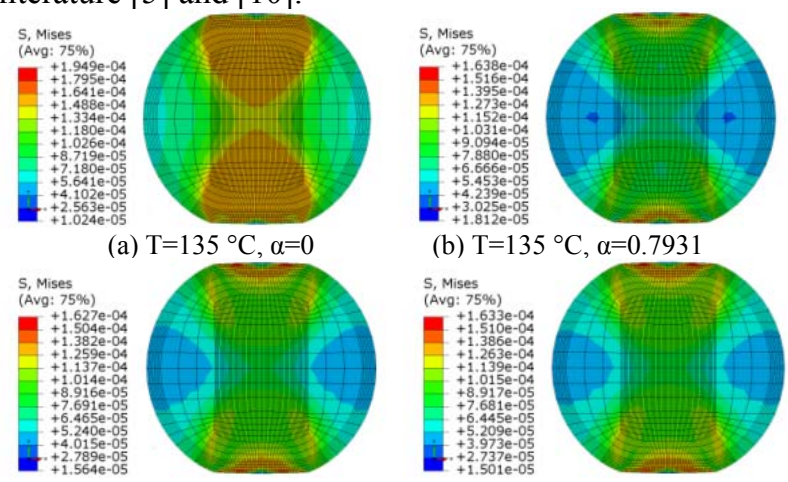

(c) $\mathrm{T}=135^{\circ} \mathrm{C}, \alpha=0.9041$

(d) $\mathrm{T}=135^{\circ} \mathrm{C}, \alpha=0.9404$

Figure 5. The effect of different curing degree on the conductive particles rebound

\section{B. The influence of curing temperature of conductive particles rebound}

Conductive adhesive under different temperature and time of the combination, can get the same curing degree. In the initial stage, conductive particles under the same curing pressure forming, as shown in Fig. 6(a). When the load of different curing temperature, curing resin will be in 
different curing rate, removed the pressure after natural cooling, the deformation of conductive particles and the interconnection conductive adhesive performance will vary. Fig. 6 (b), (c) and (d) shows the stress diagram of conductive particles after curing temperature is cooled to room temperature, and the curing degree is 0.85 .

Contrast Fig. 5(a) and Fig. 6(a), The rise of temperature makes the stress and strain of conductive particles increased. According to the simplified mechanics model Fig. 1, When the curing degree is the same, the effect of cured resin hinder conductive particles rebound is the same; Raise the temperature at the same time makes the deformation of conductive particles and back stretch of conductive particles $F_{s}$ increased. But in the process of rebound, the increase of the $F_{s}$ can lead to reduce the amount of deformation of the conductive particles instead. Through the simulation calculation, As shown in table 2, increase the contact area first, and then gradually decrease as the curing temperature increases, and the residual stress of internet interface is the same.

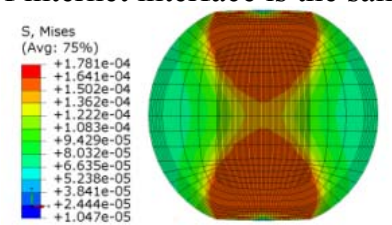

(a) $\mathrm{T}=25^{\circ} \mathrm{C}, \mathrm{t}=0 \mathrm{~s}$

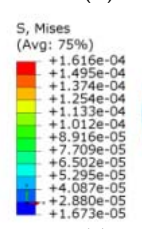

(c) $\mathrm{T}=135^{\circ} \mathrm{C}, \mathrm{t}=402.2 \mathrm{~s}$

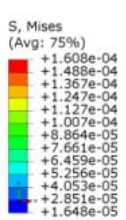

(b)
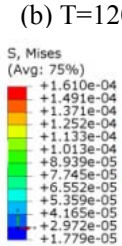

(d) $\mathrm{T}=150{ }^{\circ} \mathrm{C}, \alpha=228.6 \mathrm{~s}$
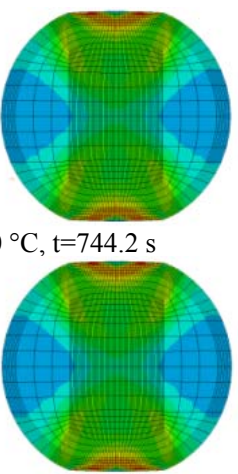

Figure 6. The effect of different temperature on the conductive particles rebound

TABLE II. THE VALUES OF SIMULATION CALCULATION

\begin{tabular}{|c|c|c|c|}
\hline & \multicolumn{3}{|c|}{$\begin{array}{c}\text { Contact Area } \\
{\left[\mathbf{u m}^{2}\right]}\end{array}$} \\
\hline & 0.80 & 0.85 & 0.90 \\
\hline $120^{\circ} \mathrm{C}$ & 12.5603 & 12.5608 & 12.5612 \\
\hline $135^{\circ} \mathrm{C}$ & 12.5589 & 12.5594 & 12.5599 \\
\hline \multirow[t]{3}{*}{$150^{\circ} \mathrm{C}$} & 12.5605 & 12.5609 & 12.5613 \\
\hline & \multicolumn{3}{|c|}{$\begin{array}{c}\text { Equivalent Von Mises Stress } \\
\text { [MPa] }\end{array}$} \\
\hline & 0.80 & 0.85 & 0.90 \\
\hline $120^{\circ} \mathrm{C}$ & 20.1076 & 20.7101 & 21.1299 \\
\hline $135^{\circ} \mathrm{C}$ & 20.0614 & 20.6939 & 21.1167 \\
\hline $150^{\circ} \mathrm{C}$ & 20.1812 & 20.8087 & 21.2145 \\
\hline
\end{tabular}

\section{SUMMARY}

The nonlinear properties of elastic modulus of the resin in relation to curing degree of the resin was defined through the phenomenological model, and then ABAQUS software is used to get the influence rule of curing temperature and curing degree on the properties of conductive adhesive interconnection. Therefore, the curing degree increased is able to increase the contact area of conductive particles so as to reduce the contact resistance, but at the same time, the interconnection interface residual stress also increased, thus easy to cause the stratification. So we need to reasonable selection of curing process to obtain the best curing degree. However, when the curing degree is determined, the contact area increased firstly, and then gradually decrease as the curing temperature increases, and when used in curing at lower temperature, the curing time increased accordingly, it will influence the economic benefits. In order to better determine the curing process parameters we need to do further research, only synthesizing electrical conductivity and mechanical properties can obtain the best curing technology parameters, and we will continue to further study.

\section{ACKNOWLEDGMENT}

The research work was financially supported by National Defense Basic Scientific Research Project"***Function Layer Interface Matching Rule and Multiple Energy Coordinated Control Method " and Guangxi Key Laboratory of Open Subject (Number: GuiKeNeng 13051-09-011K).

\section{REFERENCES}

[1] Mengjiang Xing, "A modeling and study of RF passive components based on LTCC technology". Xian: Xidian University, 2012 .

[2] Bai N, Chen X, Gao H, "Simulation of uniaxial tensile properties for lead-free solders with modified Anand mode". Materials \& Design, vol. 30, no. 1, 2009, pp.122-128.

[3] George E, Das D, Osterman M, et al, "Thermal cycling reliability of lead-free solders (SAC305 and Sn3.5Ag) for high-temperature applications". Device and Materials Reliability, IEEE Trans, vol. 11 , no. 2, 2011, pp.328-338.

[4] Zhengiia Wang, "Analysis of anisotropic conductive adhesive interconnecting under multi-factors influence and optimization for its performance". Wuhan: Huazhong University of Science \& Technology, 2010.

[5] Chin M, Barber J R, Hu S J, "Effect of elastic recovery on the electrical contact resistance in anisotropic conductive adhesive assemblies". Components and Packaging Technologies, IEEE Trans, vol. 29, no. 1, 2006, pp.137-144.

[6] Rong Z, Huang F, Shen X, et al, "Preparation and properties of dipropargyl ether of bisphenol A-modified bismaleimide resins and composites". Polymer composites, vol. 29, no. 5, 2008, pp.483-488.

[7] Xiaoxia Wang, "Numerical simulation of curing deformation of thermosetting resin matrix composites". Shangdong: Shangdong University, 2010.

[8] Haiqing Jiang, Yu Yan, Xiaojun Wang,et al, "The curing kinetics of unsaturated polyester resin/sediment system studied by dynam ic dsc". Fiber reinforced plastics/Composites,vol. 18, no. 4, 2010, pp. 33-36.

[9] Bogetti, T. A., \& Gillespie, J. W, "Process-induced stress and deformation in thick-section thermoset composite laminates". Journal of Composite Materials, vol. 26, no. 5, 1992, pp.626-660.

[10] Jong W R, Peng S H, Tsai H C, "Characteristics of a new-type anisotropic conductive film joints during the bonding process". International Communications in Heat and Mass Transfer, vol. 37, no. 5, 2010, pp.506-513. 